\title{
Electric Power Steering Power Circuit Health Assessment and Mitigation Strategy
}

\author{
Wen-Chiao Lin ${ }^{1}$, Graeme Garner ${ }^{2}$, Yat-Chung Sunny Tang ${ }^{3}$, and Arash Mohtat*4 \\ ${ }^{1}$ General Motors Global R\&D, Warren, MI, 48092, USA \\ wen-chiao.lin@gm.com \\ ${ }^{2}$ General Motors Company, Canadian Technical Centre, Markham, Ontario, L3R 4H8, Canada \\ graeme.garner@gm.com \\ ${ }^{3}$ General Motors Global Product Development, Warren, MI, 48092, USA \\ yat-chung.tang@gm.com \\ ${ }^{4}$ Invision AI, Toronto, Ontario, Canada \\ arash.mohtat@invision.ai
}

\begin{abstract}
With recent developments of energy efficient design and control for electric motors, electrical subsystems and components have become integral parts of main actuators in vehicle systems (e.g., steering and propulsion systems). To ensure proper vehicle operations, it is important to make sure that electrical power is properly transmitted through the power circuit from vehicle power source to the electric motor. However, degradation in the power circuit health, which often manifests itself as increased resistance, may affect power transmission and degrade the system performance. For example, in Electric Power Steering (EPS) systems, if the EPS power circuit resistance is increased and the EPS is drawing power to assist the driver, voltage at the EPS module will drop significantly, causing the EPS to reset and, consequently, Loss of Assist (LOA) incidents. As a result, it may suddenly become very hard to steer the vehicle. While previous work has partially addressed this issue by developing algorithms that estimate resistance increase in EPS power circuits, this paper further validates and refines the algorithms for vehicle on-board and off-board implementations using test drive data collected. Since on- and off-board implementations impose different limits on signal sampling rates, a total of 250 and 465 minutes of data are respectively collected with various vehicle speeds and steering maneuvers. Moreover, a fault mitigation strategy, referred to as EPS Anti-Loss-of-

\footnotetext{
Wen-Chiao Lin et al. This is an open-access article distributed under the terms of the Creative Commons Attribution 3.0 United States License, which permits unrestricted use, distribution, and reproduction in any medium, provided the original author and source are credited.

*Work documented in this paper was conducted while A. Mohtat was at General Motors Canadian Technical Centre.
}

Assist (ALOA), is proposed that gradually and proactively reduces EPS torque assist as resistance in the EPS power circuit increases so that the EPS voltage is kept above a resetting threshold. Stationary steering tests and demonstrations on parking lot maneuvers are conducted, which show that, with the proposed fault mitigation strategy, negative effects of increased EPS power circuit resistance can be mitigated without noticeable changes in normal driving experience.

\section{INTRODUCTION}

\subsection{Motivation}

With increasing awareness of power consumption and fuel economy, electric motors are increasingly being employed to replace or augment traditional chassis actuators that consume more power (Denton, 2004). For example, Electric Power Steering (EPS) Systems (Badawy, Bolourchi, \& Gaut, 1997; Burton, 2002) replace most hydraulic steering systems in vehicles ranging from compact cars to light duty trucks (Eki, Teratani, \& Iwasaki, 2007). Although electric motors may not be able to provide enough steering assist in heavy duty trucks, they may be used to augment hydraulic power steering systems for efficiency (Morton, Spargo, \& Pickert, 2014). Similarly, an electric motor may be included in a brake system to increase brake effort (Hwang \& Kwon, 2019). To ensure such systems work properly, it is important to make sure that electrical power is properly transmitted through the power circuit from vehicle power source to the electric motor. However, degradation in the power circuit health, which often manifests itself as increased resistance, may affect power transmission and degrade the system performance. In this pa- 
per, we focus on EPS systems due to its direct influence on vehicle dynamics and lateral control. However, general ideas presented here may be applied to other electric components.

Consider Figure 1, where an EPS is connected to a power source providing voltage $V_{B}, V_{C}$ and $V_{M}$ are the voltages seen by the EPS ECU from the power source loop and the electric motor, respectively, $I_{C}$ and $I_{M}$ are the currents passing through the power connector and the motor, respectively, $R_{M}$ is the motor internal resistance, $\omega$ is the EPS motor speed, $n$ is the gear ratio between the EPS motor and steering rack pinion, $\dot{\theta}_{p}$ is the pinion angular velocity, and $T$ is the assist torque provided by the EPS motor. The connection

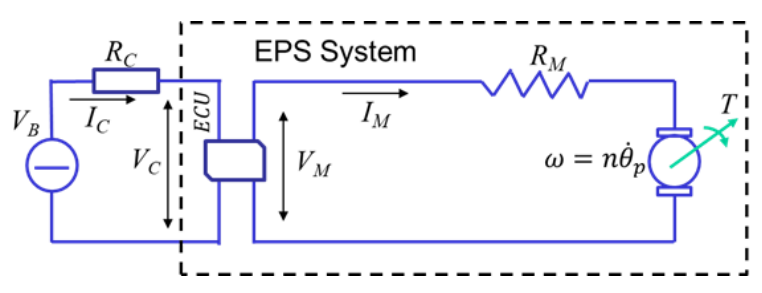

Figure 1. EPS connected to power source.

between the EPS and power source includes power wires and connectors, which may experience corrosion or other types of faults and failures over time. The resistance $R_{C}$ models the increased resistance due to degraded health of power circuit (e.g., corroded connector). In conventional driving, the EPS motor will provide additional torque to assist the driver, drawing power (and current) from the power supply. The amount of torque provided is based on the vehicle speed, driver input torque, and other factors, depending on supplier implementation. If EPS is trying to provide a significant amount of assist (power / torque) to the driver, the current drawn from the EPS, $I_{C}$, will be very large. If $R_{C}$ is increased due to poor EPS power circuit health, a significant voltage drop will be experienced at the EPS end. If the voltage is dropped below a resetting threshold, the EPS, by design, will reset and suddenly stop providing the assist torque. As a result, it will suddenly become very difficult to steer the vehicle, and this is commonly referred to as a Loss of Assist (LOA) incident. The main goal of this paper is to develop solutions that not only assess EPS power circuit health by estimating increase in its resistance but also mitigate the negative effect of this increase.

\subsection{Previous Work}

This subsection reviews related work on electrical connectivity fault diagnosis as well as fault diagnosis and prognosis (D\&P) for EPS systems.

As reviewed in (Du, Nagose, Bloom, \& Julson, 2017), various techniques to diagnose electrical connectivity issues have been developed over the years (Chung, Amarnath, \& Furse, 2009; Chung, Furse, \& Pruitt, 2005; Furse et al., 2003; Furse,
Chung, Lo, \& Pendalaya, 2006; Okada et al., 2015; Shi \& Kanoun, 2014; Smail, Hacib, Pichon, \& Loete, 2011; Smail, Pichon, Olivas, Auzanneau, \& Lambert, 2010; Smith, Furse, \& Gunther, 2005; Tsai, Lo, Chung, \& Furse, 2005; Will \& Rolfes, 2013). These techniques detect whether a wire becomes open, a connector is disconnected, or a wire is shorted to the ground. For example, (Chung et al., 2009) detects these issues by measuring properties such as inductance, resistance, conductance, capacitance or impedance. References (Will \& Rolfes, 2013; Furse et al., 2006; Shi \& Kanoun, 2014; Smail et al., 2010, 2011; Okada et al., 2015) measure characteristics of transmission or reflection signals generated from active or passive electrical signals through the circuit to detect connectivity issues. Two fundamental techniques have been developed, namely time-domain transmissometry (TDT) and time-domain reflectometry (TDR). TDT (Will \& Rolfes, 2013) monitors the transmission characteristics of an electrical signal to determine the fault severity, while TDR (Furse et al., 2006; Shi \& Kanoun, 2014; Smail et al., 2010, 2011; Okada et al., 2015) measures the amplitude and the timing of the reflected signal to determine the location and the type of the fault. Techniques using frequency-domain characteristics to detect faults in electrical connectivity, referred to as frequency-domain transmissometry (FDT) or frequencydomain reflectometry (FDR) (Furse et al., 2003; Chung et al., 2005; Tsai et al., 2005), have also been developed. While previous work focuses on electrical connection open and short issues, this paper, however, focuses on estimating resistance increase in the power circuit caused by, e.g., corrosion, and subsequent mitigation actions.

As the EPS system is a main actuator for vehicle lateral control, fault D\&P algorithms have been developed in previous research to address potential faults / failures (Lin \& Ghoneim, 2016; Lin \& Du, 2018; Mohtat, Garner, Lin, \& Mehrabi, 2020). In particular, (Lin \& Ghoneim, 2016) developed methods to monitor the EPS motor health through parameter estimation techniques as well as an algorithm to detect increase in steering system friction, which is validated and refined in (Mohtat et al., 2020). Reference (Lin \& Du, 2018) provide a canary-based method to detect loose connections in the EPS power circuit as well as a preliminary algorithm to estimate resistance increase in the circuit. Using test vehicle data, this paper validates and refines the previously developed EPS power circuit resistance estimation algorithm (Lin \& Du, 2018) for on- and off-board implementations. Furthermore, using the estimated resistance, a strategy to proactively mitigate the negative effect of increased resistance is developed. In particular, a supervisory controller concept that includes EPS power circuit health assessment (resistance estimation) and a strategy to mitigate the negative effects of steering LOA described in Section 1.1 is developed. 


\subsection{Outline of Paper}

The rest of the paper is organized as follows. Section 2 develops the supervisory controller concept to estimate EPS power circuit resistance and to mitigate LOA incidents due to resistance increase, while Section 3 describes the test vehicle setup. Section 4 reviews the EPS power circuit resistance estimation algorithm in ( $\mathrm{Lin} \& \mathrm{Du}, 2018)$, describes the test data collected, and validates and refines the algorithm for onand off-board implementations. Section 5 describes the strategy to mitigate the negative effects of power circuit resistance increase, stationary testing of the strategy, and demonstration of it on parking lot maneuvers. Conclusions are given in Section 6, and acknowledgement of assistance for this work is given after the conclusion section. Driving maneuvers and noise factors considered for the test data collection are described in the Appendix.

\section{2. eps Power Circuit health Assessment and FAUlt Mitigation FrameWORK}

The framework for EPS power circuit health assessment and mitigation considered in this paper is shown in Figure 2, where the supervisory controller consists of a health assessment and a mitigation module. The health assessment module

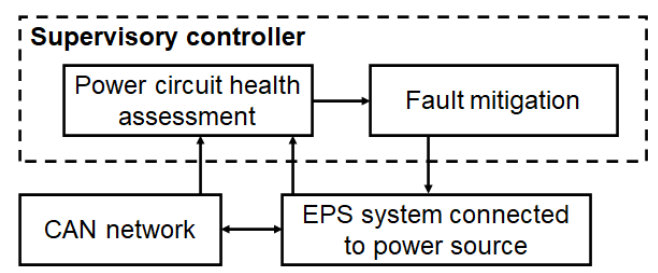

Figure 2. Proposed supervisory controller.

computes the health of the EPS power circuit by estimating the resistance in the circuit. Inputs to this module are signals necessary for this computation from the EPS system and other vehicle subsystems via the vehicle control area network (CAN) bus. The health assessment results, e.g., the estimated resistance, is then used by the mitigation strategy to avoid negative effects of steering LOA as the resistance increases. While (Lin \& $\mathrm{Du}, 2018$ ) has developed an algorithm to estimate the EPS power circuit resistance, Section 4 validates and refines these algorithms for on- and off-board implementations. Section 5 describes the fault mitigation strategy to avoid steering LOA.

\section{Test Vehicle Setup}

A test vehicle is instrumented to collect data for validating and refining previously developed EPS power circuit resistance estimation algorithms for on- and off-board implementations. Furthermore, it is also used to test and demonstrate efficacy of developed fault mitigation strategy. In particular, as Figure 3 illustrates, adjustable (variable) power resistors are installed in series between the vehicle power source and the EPS system of the test vehicle. The power resistors em-

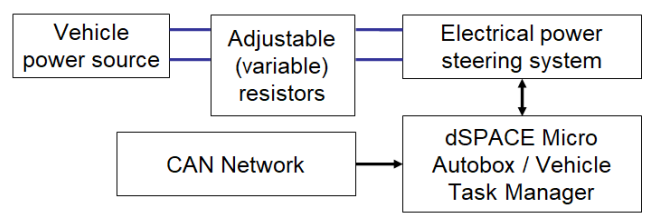

Figure 3. Test vehicle setup.

ulate power circuit health degradation that manifest itself as increased electrical resistances, and the amount of resistance introduced can be selected as needed. For example, in validating and refining the power resistance estimation algorithm, data was collected at four resistance levels with the labels and injected resistances shown in Table 1. As Table 1 indicates,

Table 1. Resistance fault injection labels and values.

\begin{tabular}{|l|c|c|}
\hline Fault level & $\begin{array}{c}\text { Injected } \\
\text { resistance }(\mathrm{m} \Omega)\end{array}$ & $\begin{array}{c}\text { Total } \\
\text { resistance }(\mathrm{m} \Omega)\end{array}$ \\
\hline Healthy $(\mathrm{H})$ & 0 & 20 \\
\hline R1 & 20 & 40 \\
\hline R2 & 40 & 60 \\
\hline R3 & 60 & 80 \\
\hline
\end{tabular}

the nominal (healthy) resistance of the test vehicle EPS power circuit is $20 \mathrm{~m} \Omega$ and the variable resistor adds resistance to the power circuit.

For on-board implementation of the EPS power circuit estimation algorithm, a dSPACE Micro-Autobox ${ }^{1}$ (MAbox) is used to host the algorithm and to collect data from the EPS and vehicle CAN bus as inputs to the algorithm. It also hosts the fault mitigation algorithm and sends commands to the EPS system. For off-board implementation of the resistance estimation algorithm, a Vehicle Task Manager (VTM) is used to collect and transmit data for off-board processing. Advantages of on-board implementation include higher sampling rates for accurate resistance estimations, while disadvantages include the computational resources required on the EPS electronic control unit (ECU). Conversely, off-board implementation does not require the additional computational resources but lower sampling rates reduces the estimation accuracy. Since off-board implementation of the resistance estimation algorithm is much less accurate than its on-board implementation (see Section 4.4), off-board implementation of fault mitigation algorithms is not considered here.

\section{EPS Power Circuit Health Assessment}

This section describes the power circuit health assessment module in Figure 2. In particular, the EPS power circuit resis-

\footnotetext{
${ }^{1}$ The Micro-Autobox is a product of dSPACE GmbH, and details of it can be obtained from https://www.dspace.com/
} 
tance estimation algorithm developed in (Lin \& Du, 2018) is validated and refined for on- and off-board implementations.

\subsection{Resistance Estimation Algorithm}

We first review the resistance estimation algorithm developed in (Lin \& Du, 2018). Referring to Figure 1, the goal of the algorithm is to estimate and detect increase in $R_{C}$, the total resistance in the power circuit between the vehicle power source and the EPS system. A quick inspection of Figure 1 indicates that the resistance $R_{C}$ can be estimated using the equation $R_{C}=\frac{V_{B}-V_{C}}{I_{C}}$. However, in typical production vehicles, there is no sensor to read the current through the power circuit $\left(I_{C}\right)$. Fortunately, this current can be approximated using other sensor measurements. Referring again to Figure 1, power conservation is applied across the EPS ECU:

$$
V_{C} I_{C}=V_{M} I_{M}
$$

where, as described in Section 1.1, $V_{C}$ and $V_{M}$ are the voltages seen by the EPS ECU from the power source loop and the electric motor, respectively, and $I_{C}$ and $I_{M}$ are the currents passing through the power connector and the motor, respectively. Noting that the power input to the motor circuit is equal to the power output, the right-hand side of the above equation can be expanded as:

$$
V_{C} I_{C}=I_{M}^{2} R_{M}+n T \dot{\theta}_{p}+P_{L},
$$

where, as mentioned in Section 1.1, $R_{M}$ is the internal resistance of the motor, $T$ is the motor's torque, $n \dot{\theta}_{p}$ is the angular speed of the motor's shaft, and $P_{L}$ is the power loss due to ECU consumption and other noises. Assuming $P_{L}$ to be negligible when $I_{M}$ is large, the above equation can be used to estimate the current through the connector, i.e., $I_{C}$. Finally, the equivalent connector resistance is estimated by applying Ohm's Law:

$$
\hat{R}_{c}=\frac{\Delta V}{I_{C}}=\frac{V_{B}-V_{C}}{\frac{I_{M}^{2} R_{M}+n T \dot{\theta}_{p}}{V_{C}}} .
$$

The quality of the estimate can be improved using batch calculations. Instead of calculating $\hat{R}_{C}$ sample-wise (i.e., using signals from a single time step and applying Eq. (3)), a leastsquares estimate is obtained by collecting a buffer of $B$ samples and applying Eq. (4) below. This equation is derived by applying ordinary least squares minimization:

$$
\begin{array}{ll}
\text { Objective: } & \text { minimize } \sum_{i=1}^{B}\left(\Delta V_{i}-R_{C} I_{C, i}\right)^{2} \quad \text { over } R_{C} \\
& \Rightarrow 0=\frac{d}{d R_{C}}\left(\sum_{i=1}^{B}\left(\Delta V_{i}-R_{C} I_{C, i}\right)^{2}\right) \\
& \Rightarrow \hat{R}_{C}=\frac{\sum_{i=1}^{B} \Delta V_{i} I_{C, i}}{\sum_{i=1}^{B}\left(I_{C, i}\right)^{2}}
\end{array}
$$

Enabling criteria are applied to ensure calculation only includes data from when the EPS motor is sufficiently activated for the minimal-loss assumption, i.e., negligible $P_{L}$, to hold. The objective of tuning the algorithm, i.e., choosing batch size and enabling conditions, is to reduce the minimum detectable increase in resistance as much as possible.

\begin{tabular}{|c|c|c|}
\hline System & Acquisition rate & Purpose \\
\hline $\begin{array}{l}\text { dSpace } \\
\text { Micro Autobox }\end{array}$ & $100 \mathrm{~Hz}$ & $\begin{array}{c}\text { On-board } \\
\text { implementation }\end{array}$ \\
\hline $\begin{array}{l}\text { Vehicle Task } \\
\text { Manager (VTM) }\end{array}$ & $\sim 1 \mathrm{~Hz}$ & $\begin{array}{c}\text { Off-board } \\
\text { implementation }\end{array}$ \\
\hline
\end{tabular}

\subsection{Test Data Collected}

Test data with the injected faults described in Table 1 are collected. Two data acquisition systems are utilized for collection, summarized in Table 2 below. Both acquisition sys-

Table 2. Data acquisition systems.

tems record signals (vehicle speed, lateral acceleration, steering angle and gradient, yaw rate, battery voltage, driver input torque, EPS motor torques, and EPS motor current and voltage) needed for the algorithm. The algorithm is implemented for both data sources to gauge its performances in on- and off-board implementations. Although both acquisition systems are collecting the same data, the VTM data presents two challenges that the MABox does not. First, since the acquisition rate is lower, it is more time-consuming to collect the needed amount of data for robust algorithm refinement. Second, the VTM data collection process introduces latency between collected signals. New signal requests are not sent until the previous signal has been received, introducing a delay on the order of one hundred milliseconds between each signal.

A variety of steering maneuvers are recorded at all health states described in Table 1, including spirals, turns, lane changes, banks, sinusoidals, and organic driving. Noise factors such as tire type, tire pressure, passenger weight, and battery load are also varied from test-to-test. Detailed descriptions of each test maneuver and noise factor are included in the Appendix. A total of 102 individual tests are conducted, summarized in Table 3. The data for each health state is split

Table 3. Resistance algorithm data collection.

\begin{tabular}{|l|c|c|c|}
\hline Health state & $\begin{array}{c}\text { Change } \\
\text { (vs. Healthy) }\end{array}$ & $\begin{array}{c}\text { MABox } \\
\text { data set (min) }\end{array}$ & $\begin{array}{c}\text { VTM data } \\
\text { (min) }\end{array}$ \\
\hline Healthy vehicle & NA & 136 & 119 \\
\hline Resistance: R1 & $+20 \mathrm{~m} \Omega$ & 72 & 117 \\
\hline Resistance: R2 & $+40 \mathrm{~m} \Omega$ & 72 & 110 \\
\hline Resistance: R3 & $+60 \mathrm{~m} \Omega$ & 70 & 119 \\
\hline
\end{tabular}

into three categories - development, validation, and test. Development data are used for experimenting and refining the algorithms, validation data are used for unbiased assessment 
of changes made during refinement and calibration of tuning parameters (batch size and enabling conditions), and test data are used for final assessment of algorithm performance. The data are distributed approximately $60 \%$ to development and $20 \%$ each to validation and testing.

\subsection{On-board Implementation}

The resistance estimation presented by Eq. (4) is implemented on both MABox and VTM data. The end-to-end algorithm is summarized in Figure 4, where the output determines whether there is resistance increase (Faulty) or not (Healthy). The enabling conditions and batch size are chosen following rigorous exploration of performance tradeoffs. The chosen enabling conditions are the minimum to ensure that the EPS system is active enough to assume negligible power loss in the ECU. Algorithm accuracy improves with batch size at the expense of increasing time-to-detection (T2D). Batch sizes larger than 80 offered slightly improved accuracy with significantly longer T2D. The development set is used to experiment with these parameters, and the validation set is used to confirm the decision.

Figures 5 and 6 show the performance of the resistance health indicator (i.e., the estimated resistance) on the MAbox test set (i.e., on data that was not used in algorithm development). In particular, Figure 5 shows the realized health indicator values vs the ground-truth health state. Each point is the health indicator output from one batch of data. The ROC curve in Figure 6 shows the classifier performance for separating healthy from all faulty health states (i.e., the performance of detecting a $>20 \mathrm{~m} \Omega$ increase) in blue, and the performance of separating healthy from $\mathrm{R} 2$ and $\mathrm{R} 3$ (i.e., detecting a $>40 \mathrm{~m} \Omega$ increase) in red. Tables 4 and 5 show improvement in results from maturing the decision. Specifically, Table 4 shows the

Table 4. Confusion matrix pre-maturation for on-board implementation.

\begin{tabular}{|c|c|c|c|c|}
\hline \multirow{2}{*}{} & \multicolumn{4}{|c|}{ Ground truth health state } \\
\cline { 2 - 5 } & H & R1 & R2 & R3 \\
\hline $\begin{array}{c}\text { Output } \\
\text { Healthy }\end{array}$ & $\mathbf{8 5}$ & 11 & 1 & 0 \\
\hline $\begin{array}{c}\text { Output } \\
\text { Faulty }\end{array}$ & 8 & $\mathbf{2 0 6}$ & $\mathbf{2 9 9}$ & $\mathbf{3 6 5}$ \\
\hline
\end{tabular}

Table 5. Confusion matrix post-maturation for on-board implementation.

\begin{tabular}{|c|c|c|c|c|}
\hline \multirow{2}{*}{} & \multicolumn{4}{|c|}{ Ground truth health state } \\
\cline { 2 - 5 } & H & R1 & R2 & R3 \\
\hline $\begin{array}{c}\text { Output } \\
\text { Healthy }\end{array}$ & $\mathbf{8 4}$ & 3 & 0 & 0 \\
\hline $\begin{array}{c}\text { Output } \\
\text { Faulty }\end{array}$ & 0 & $\mathbf{2 0 5}$ & $\mathbf{2 9 1}$ & $\mathbf{3 5 6}$ \\
\hline
\end{tabular}

confusion matrix for the results pre-maturation, while Table 5 shows the confusion matrix after applying an " $X$ for $Y$ " maturation strategy with parameters $X=7$ and $Y=10$. This maturation strategy considers the $Y$ most recent results to yield a more accurate determination of health, where $Y$ is a positive integer. A matured sampled is labelled as faulty only if at least $X$ of the most recent $Y$ results are faulty, otherwise it is labelled as healthy. Larger values of $Y$ tend to yield more accurate diagnostics at the cost of longer time to detection given the need for more results before issuing matured output. Smaller values of $X$ will favor issuing a positive (faulty) output (prone to false positives), and larger values of $X$ will favor issuing a negative (healthy) output (prone to false negatives). These integer parameters can be optimized by exhaustive search of complexity $O\left(\sum_{n=1}^{N} n\right)$, where $N$ is the maximum value considered for $Y$. In the current study, values of $X=7$ and $Y=10$ were found to yield no false positives and high true positive rates (i.e., low false negative rates), while making decisions in a reasonable amount of time, on the development data.

The algorithm performs very well after maturation, with $0 \%$ false positive rate (FPR) and 99\% true positive rate (TPR), with false negatives only occurring at the R1 $(+20 \mathrm{~m} \Omega)$ fault level. When this algorithm was run on organic driving data (i.e. not constructed maneuvers but natural driving), it took an average of 12 steering events to reach a matured decision.

A natural extension to the above binary diagnostic approach (i.e., output either healthy or faulty) is to explore whether the estimated resistance could be used as a health stage estimate. Table 6 shows the raw (unmatured) results for a multiclass classification applied to the development data. These results were derived using a three-threshold classifier to define the four output classes, in which the three thresholds were tuned to minimize misclassifications. The resulting accuracy is $81 \%$, which could be improved by employing a multi-class maturation strategy.

Table 6. Multi-class confusion matrix for on-board implementation.

\begin{tabular}{|c|c|c|c|c|}
\hline \multirow{2}{*}{ Predicted health state } & \multicolumn{5}{|c|}{ Ground truth health state } \\
\cline { 2 - 5 } & H & R1 & R2 & R3 \\
\hline H & $\mathbf{9 0}$ & 29 & 1 & 1 \\
\hline R1 & 2 & $\mathbf{1 6 3}$ & 1 & 1 \\
\hline R2 & 1 & 29 & $\mathbf{2 3 6}$ & 61 \\
\hline R3 & 0 & 29 & 32 & $\mathbf{2 9 8}$ \\
\hline
\end{tabular}

\subsection{Off-board Implementation}

When the resistance detection algorithm is adapted to VTM data, the performance declined significantly. The batch size has to be reduced from 80 to 12 to accommodate for the significantly reduced quantity of available data. This batch reduction, combined with latency in the VTM data collection 


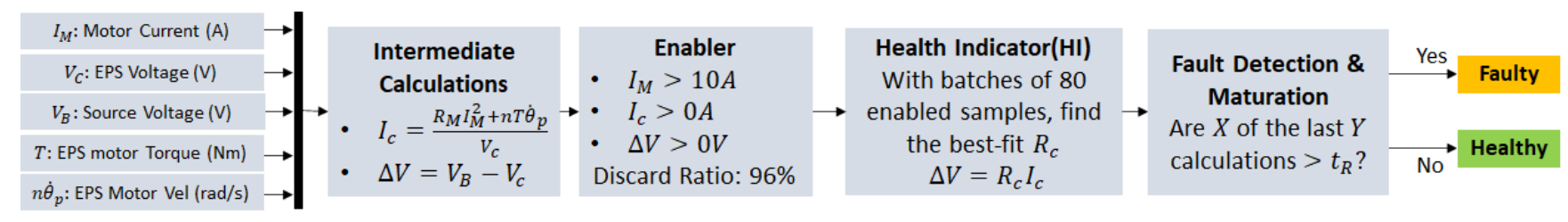

Figure 4. End-to-end resistance estimation / increase detection algorithm.

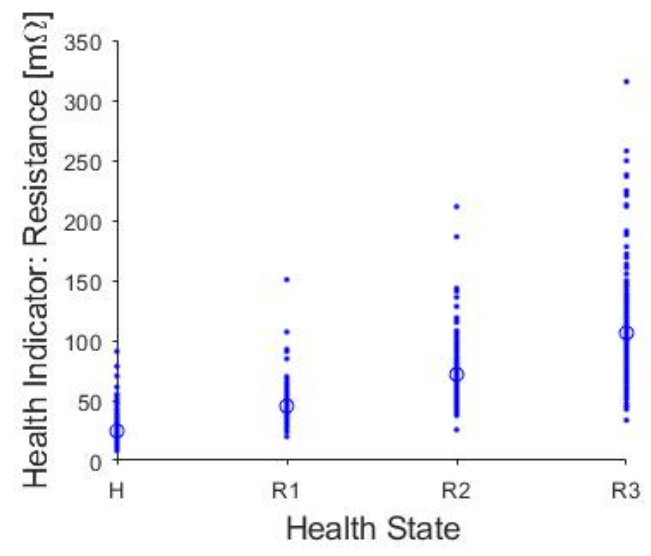

Figure 5. Estimated resistance for on-board implementation.

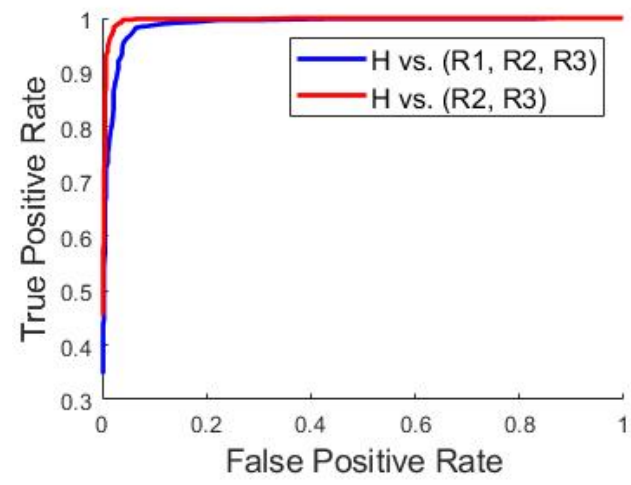

Figure 6. ROC curve for on-board resistance classification.

method, are likely the two most significant factors in the degraded performance.

Various data-driven approaches to detecting a resistance increase are experimented with to improve the performance using VTM data. The best performance comes from extending the regression estimate from a one degree-of-freedom (DOF) approach to a two DOF approach. With each batch of voltage $(\Delta V)$ and current $\left(I_{c}\right)$ data, the least-squares slope $\left(R_{C}\right)$ and intercept $\left(V_{L}\right)$ are calculated using Eq. (5). The voltagelike intercept term $V_{L}$ captures power loss $P_{L}$ within the EPS ECU (that was assumed negligible previously). The deriva- tion follows an ordinary least-squares approach:

$$
\begin{gathered}
\text { Objective: minimize } \sum_{i=1}^{B}\left(\Delta V_{i}-\left(R_{C} I_{C, i}-V_{L}\right)\right)^{2} \\
\text { over } R_{C}, V_{L} \\
\Rightarrow\left[\begin{array}{c}
\frac{d}{d R_{C}}\left(\sum_{i=1}^{B}\left(\Delta V_{i}-\left(R_{C} I_{C, i}-V_{L}\right)\right)^{2}\right) \\
\frac{d}{d V_{L}}\left(\sum_{i=1}^{B}\left(\Delta V_{i}-\left(R_{C} I_{C, i}-V_{L}\right)\right)^{2}\right)
\end{array}\right]=\left[\begin{array}{l}
0 \\
0
\end{array}\right] \\
\Rightarrow\left[\begin{array}{c}
\sum_{i=1}^{B}\left(I_{C, i}\right)^{2} \sum_{i=1}^{B}\left(I_{C, i}\right) \\
\sum_{i=1}^{B}\left(I_{C, i}\right) B
\end{array}\right]\left[\begin{array}{c}
R_{C} \\
V_{L}
\end{array}\right] \\
=\left[\begin{array}{c}
\sum_{i=1}^{B} \Delta V_{i} I_{C, i} \\
\sum_{i=1}^{B} \Delta V_{i}
\end{array}\right] .
\end{gathered}
$$

A linear support vector machine (Figure 7) is then trained to classify the system as healthy or faulty based on both the intercept and slope of the current-voltage relationship. This

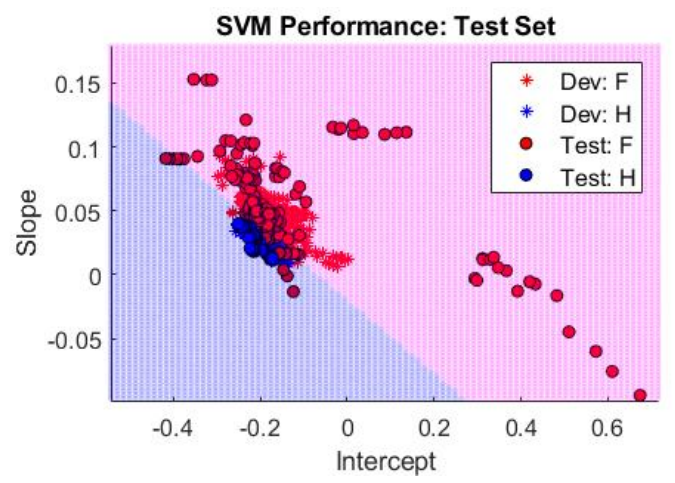

Figure 7. Classification of healthy (H) vs. faulty (R2, R3) data using a two degree of freedom model.

increased the off-board algorithm performance in classifying healthy from R2 and R3 to $99 \%$ TPR at $0 \%$ FPR after maturation strategy is applied. Although the resistance detection algorithm was able to achieve strong results for identifying 40 $\mathrm{m} \Omega$ (R2 and R3) increases in resistance, the algorithm did not exhibit as strong results as the on-board algorithm for detecting $20 \mathrm{~m} \Omega$ (R1) increases. As seen in Table 7, the R1 class $(+20 \mathrm{~m} \Omega)$ is correctly identified as faulty with a TPR of only $74 \%$. The results summarized here indicate that the on-board algorithm can accurately detect a resistance increase as small as $+20 \mathrm{~m} \Omega$, whereas the off-board algorithm is able to detect resistances increases as small as $+40 \mathrm{~m} \Omega$. 
Table 7. Confusion matrix post-maturation for off-board implementation.

\begin{tabular}{|c|c|c|c|c|}
\hline \multirow{2}{*}{} & \multicolumn{4}{|c|}{ Ground truth health state } \\
\cline { 2 - 5 } & H & R1 & R2 & R3 \\
\hline $\begin{array}{c}\text { Output } \\
\text { Healthy }\end{array}$ & $\mathbf{8 2}$ & 43 & 5 & 0 \\
\hline $\begin{array}{c}\text { Output } \\
\text { Faulty }\end{array}$ & 0 & $\mathbf{1 2 5}$ & $\mathbf{1 6 5}$ & $\mathbf{2 0 5}$ \\
\hline
\end{tabular}

Note that, although we have focused on detecting resistance increase of, e.g., 20 or $40 \mathrm{~m} \Omega$, the idea can be generalized to classifying different levels of resistance increase. The detected / classified resistance increase level can then be used in the fault mitigation strategy in Figure 2 and Section 5.

\section{Fault Mitigation}

This section describes the fault mitigation module in Figure 2 using the power circuit resistance estimation (or the detected / classified resistance increase level from Section 4) as input. Since the goal is to avoid LOA incidents due to power circuit resistance increase, the mitigation strategy is referred to as EPS Anti-Loss-of-Assist (ALOA).

\subsection{EPS Anti-Loss-of-Assist Solution}

We first review operations of a typical EPS system under nominal conditions. As shown in Figure 8, a desired torque assist value is first determined by the EPS ECU based on driver torque input, vehicle speed, and other factors. Sub-

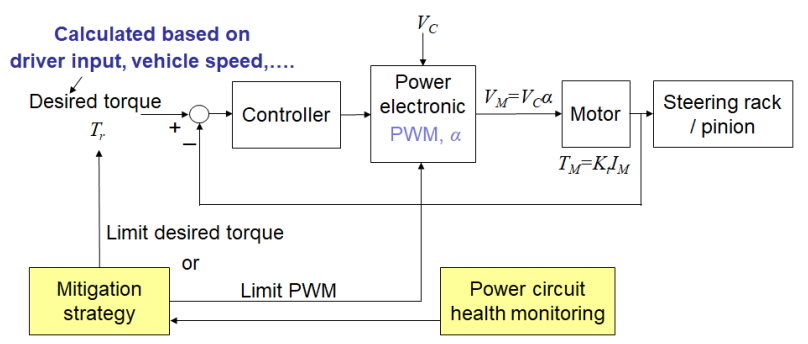

Figure 8. A possible supervisory control strategy.

sequently, a controller is designed to meet the desired torque, e.g., via feedback as shown in the figure.

As described in Section 1.1, LOA incidents result from EPS system voltage being lower than a resetting value. Hence, referring to Figure 1, the goal of EPS ALOA is to keep the voltage, $V_{C}$, at the EPS higher than a threshold value to keep the EPS system from resetting. One way to achieve this is to limit current draw, $I_{C}$, to the EPS system. While there are many ways to do this, e.g., limiting the desired torque or limiting the PWM duty cycle, the approach we take in this paper is empirical based. Specifically, we multiply the nominal desired steering assist torque by a percentage determined from a torque reduction curve and the estimated power circuit re- sistance. One example is shown in Figure 9. As the figure in-

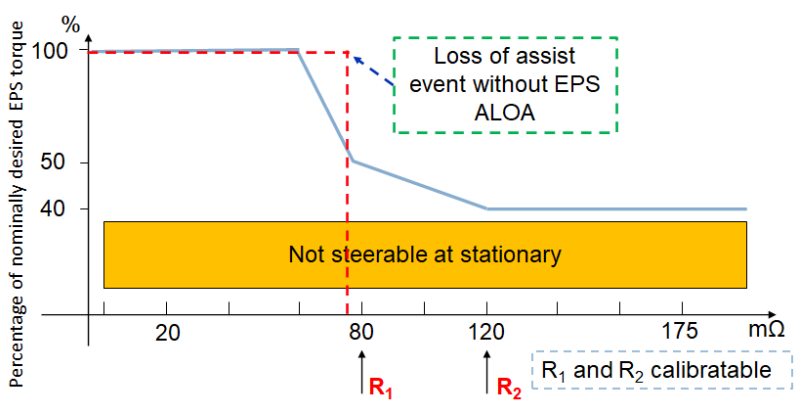

Figure 9. Torque reduction curve.

dicates, when the estimated resistance is less than $60 \mathrm{~m} \Omega$, the EPS provides the torque assist that it is nominally designed to give. As the estimated resistance increases above $60 \mathrm{~m} \Omega$, the EPS decreases the assist torque linearly down to $50 \%$ of its nominally designed value when the power circuit resistance becomes $80 \mathrm{~m} \Omega$. Afterwards, the EPS torque assist decreases linearly down to $40 \%$ of its nominally designed value until the estimated resistance becomes $120 \mathrm{~m} \Omega$. A warning may be set to notify the driver of reduced assist when torque reduction is below $50 \%$ of designed assist. In this curve, it is assumed that the vehicle is too difficult to steer with less than $40 \%$ amount if steering assist. Beyond this point, the vehicle has to be serviced. Note that the red dashed line in the figure illustrates behavior of EPS systems without reduced torque assist. Specifically, the EPS system will provide as much assist as nominally designed to the driver even when resistance in its power circuit is increasing. After the resistance is increased to a certain level, voltage at EPS system end will drop below the resetting threshold (as explained in Section 1.1) and the EPS will suddenly reset and cause an LOA incident. In contrast, the proposed approach here gradually and proactively reduces the steering assist so that the EPS voltage is kept above a resetting threshold, avoiding an LOA incident.

Note that the parameters in the torque reduction curve is highly dependent on the specific vehicle platform and should be calibrated for various steering maneuvers. Experiments based on similar vehicle platforms, however, can be used as a starting point for the calibration process of new vehicle platforms under development. Note also that in Figure 9, we assume that the resistance estimation / detection resolution is higher than the on-board implementation of the algorithm in Section 4.3, which may be possible in the future if direct measurements of $I_{C}$ become available with additional sensors.

\subsection{Stationary Testing}

Since the EPS system is designed to provide more torque assist, and hence, draw more current when the vehicle is at low speed or stationary (i.e., speed 0), we focus on vehicle testing and demonstrations for these scenarios. 
Figure 10 shows that LOA events can indeed be reduced by reducing EPS assist torque as power circuit resistance increases. In particular, in Figure 10, the horizontal axis in-

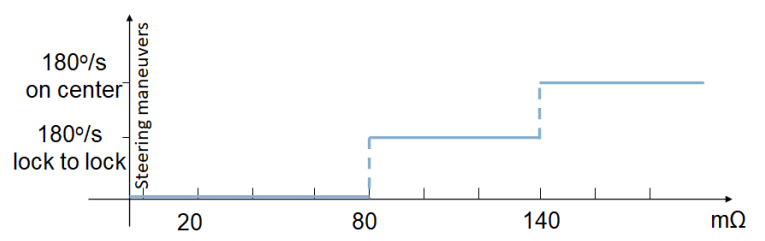

(a) EPS LOA incidents with EPS providing full assist.

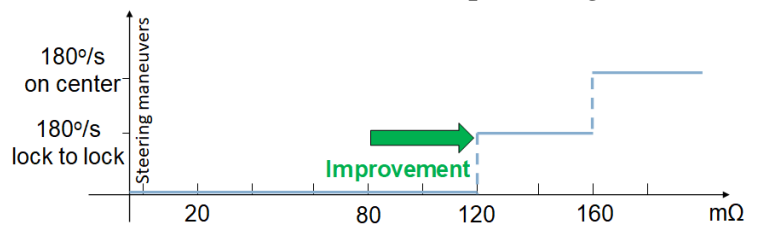

(b) EPS LOA incidents with EPS $50 \%$ assist.

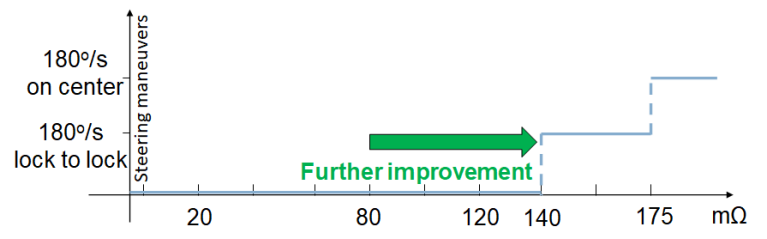

(c) EPS LOA incidents with EPS $40 \%$ assist.

Figure 10. EPS LOA incidents at different levels of assist.

dicates the EPS power circuit resistance, while the vertical axis indicates when LOA incidents start to appear. Typically, the EPS provides more assist for lock to lock steering than for on-center $\left( \pm 90^{\circ}\right)$ steering. As a result, LOA incidents occur at lower power circuit resistances for lock to lock steering. Hence, for example, in Figure 10(a), LOA events occur when the steering system is lock to lock at speed $180^{\circ} / \mathrm{s}$ and the resistance is at $80 \mathrm{~m} \Omega$, while LOA events occur when the steering system is on center at speed $180^{\circ} / \mathrm{s}$ and the resistance is at $140 \mathrm{~m} \Omega$. To illustrate the improvement, note that in Figure 10 (b), where EPS torque assist is lowered to $50 \%$ of its nominally designed value, LOA events for lock to lock steering at speed $180^{\circ} / \mathrm{s}$ do not occur until the resistance is at $120 \mathrm{~m} \Omega$. Similarly, LOA events for on center $\left( \pm 90^{\circ}\right)$ steering at speed $180^{\circ} / \mathrm{s}$ do not occur until the resistance is at $160 \mathrm{~m} \Omega$. Further improvements can be seen in Figure 10(c), where the torque assist is reduced to $40 \%$ of its nominally designed value.

Figure 11 further illustrates reduction of LOA incidents by reducing EPS torque assist when steering wheel speed is $180^{\circ} / \mathrm{s}$ lock to lock. In Figure 11, the horizontal axes indicate time in seconds, while the vertical axis in the top subfigure indicate the percentage reduction of the EPS torque assist that is being commanded. The vertical axis in the bottom sub-figure indicate occurrences of LOA incidents, i.e., when an LOA incident occurs, an indication of 1 is plotted. With this understanding, Figure 11 shows that at $80 \mathrm{~m} \Omega$ resistance,
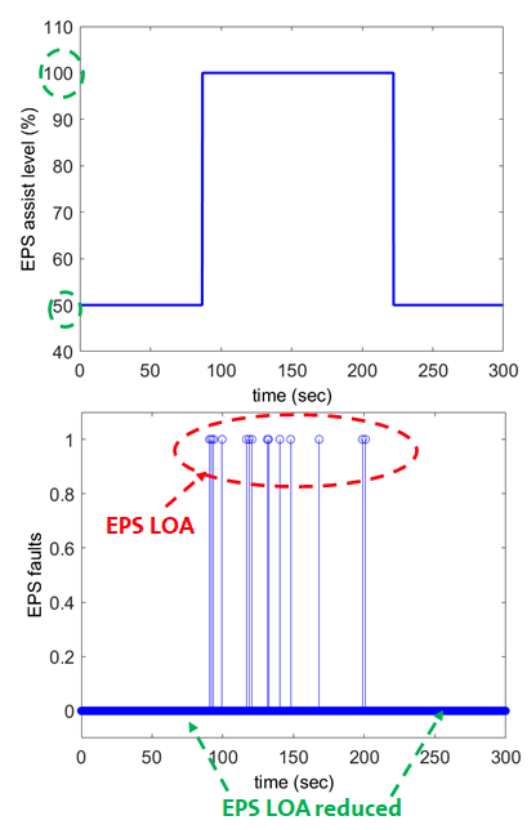

Figure 11. Reduction of LOA incidents at $80 \mathrm{~m} \Omega$ resistance.

LOA occurs when EPS is providing $100 \%$ of nominal assist, while LOA events are significantly reduced if EPS is providing $50 \%$ of nominally designed assist.

The proposed torque reduction curve shown in Figure 9 is based on this stationary test. Note that the values $R_{1}$ and $R_{2}$ in Figure 9 is platform dependent and should also be adjusted for other driving scenarios such as low speed parking maneuvers considered in Section 5.3.

\subsection{Demonstration}

Since most LOA incidents occur during parking lot maneuvers, we further illustrate that the proposed EPS ALOA strategy can significantly reduce LOA incidents by demonstrations considering three maneuvers: (i) static steering (steering wheel is steered from lock to lock (i.e., $\pm 500^{\circ}$ )); (ii) reversing out of a parking space and steering quickly to align the vehicle with the road; and (iii) suddenly turning to park a vehicle into a parking space, which may be spotted late. During the demonstration, the resistance in the EPS power circuit is set to an estimated value of $50 \mathrm{~m} \Omega$. The EPS assist torque is first set to $100 \%$ of its designed value. After the demonstrator completed the three maneuvers above, during which LOA incidents occur, the EPS assist torque is then set to $50 \%$ of its designed value. The purpose for this is to let the demonstrator feel the LOA before and after EPS assist torque reduction, and notice the reduction in LOA incidents.

Figure 12(a)-(d) plots data collected during a specific demonstration run. Referring to Figure 12(a), from time 3750s to $3950 \mathrm{~s}$, the vehicle is driven from garage to test parking 


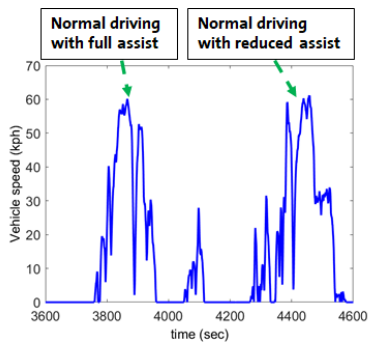

(a) Vehicle speed.

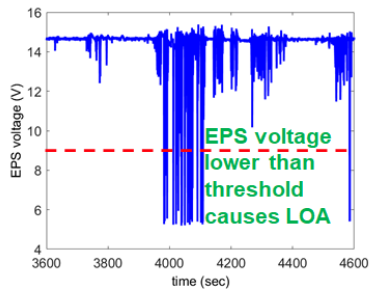

(c) EPS voltage level.
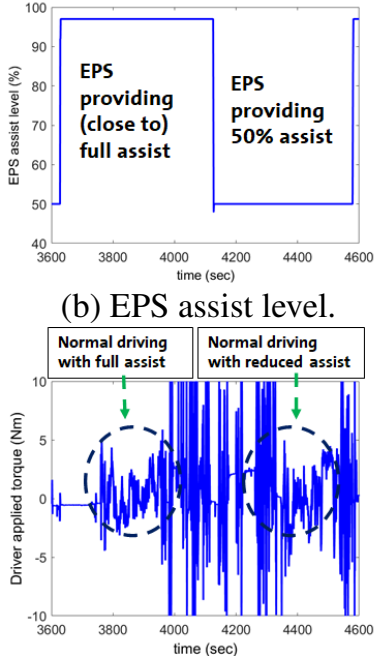

(d) Driver torque. (b) EPS assist level.

Figure 12. Data collected during demonstration.

lot with full torque assist without additional resistance introduced into the EPS power circuit. From time 3950s to 4250s, a demonstration is conducted following the maneuvers described above including some explanations and discussions with the demonstrator. From time 4250 s to 4550 s, the vehicle is driven back from test parking lot back to garage with the introduced resistance and reduced EPS torque assist. The trips back and forth from garage and test parking lot emulate normal driving on public roads. Figure 12(b) indicates the EPS assist level throughout the demonstration. In particular, from time 3600 s to 4100 s, the EPS is at its nominal assist level, while from 4100 s to 4550 s, the EPS assist is at $50 \%$ of its designed level. Figure 12(c) indicates the voltage measured at the EPS end, where the EPS resets (causing loss of assist incidents) when the voltage is below the resetting threshold. Clearly, with reduced EPS torque assist, LOA incidents are significantly reduced. Figure 12(d) plots the driver input torque throughout the demonstration. We note that the steering effort during trips back and forth from garage to test parking lot are similar for EPS with and without torque reduction. Hence, with EPS torque assist reduction, LOA incidents can be reduced, while steering effort during normal driving can more or less stay the same. An explanation for this observation is that the EPS typically provides much less assist torque in normal driving / steering scenarios than in the parking lot scenarios considered. Hence, the driver may not notice much difference when reducing the EPS assist by $50 \%$ in normal driving scenarios.

\section{Conclusion}

A supervisory controller concept for EPS systems that includes power circuit health assessment and fault mitigation modules was developed. Specifically, the health assessment module estimates the resistance increase in the EPS power circuit due to faults such as corrosion, and the fault mitigation module uses the estimation results to reduce EPS assist torque to keep the EPS system from suddenly resetting, causing LOA events. Refinement and validation of the resistance estimation algorithm using data collected from a test vehicle indicate that the resistance estimation can detect resistance increase of 20 and $40 \mathrm{~m} \Omega$ for on- and off-board implementations, respectively. As the estimated power circuit resistance increases, the fault mitigation module reduces the EPS assist effort by a torque reduction curve, which can be calibrated for different vehicle platforms and driving purposes. Testing and demonstration results indicate that LOA incidents can successfully be reduced without affecting normal driving feel. Future work includes exploring other methods of fault mitigation, e.g., limiting EPS motor controller PWM based on estimated power circuit resistances.

\section{ACKNOWLEDgment}

The authors thank Dr. Xinyu Du for initial development and discussions on EPS power circuit resistance estimation and fault mitigation strategies. Support from James Di Donato, Kerima Musanovic, Daniel Demitrish, Daniel Rudd, and Jerry Kowalski is also greatly appreciated.

\section{REFERENCES}

Badawy, A. A., Bolourchi, F., \& Gaut, S. K. (1997). Esteer system redefines steering technology. Automotive Engineering, 105(9), 15-18.

Burton, A. W. (2002). Control objectives and systems analysis: innovation drivers for electric power-assisted steering. In Proc. of the 2002 american control conference.

Chung, Y. C., Amarnath, N. N., \& Furse, C. M. (2009). Capacitance and inductance sensor circuits for detecting the lengths of open- and short-circuited wires. IEEE Transactions on Instrumentation and Measurement, 58, 2459-2502.

Chung, Y. C., Furse, C., \& Pruitt, J. (2005). Application of phase detection frequency domain reflectormetry for locating faults in an f-18 flight control harness. IEEE Transctions on Electromagnetic Compatibility, 47, 327-334.

Denton, T. (2004). Automobile electrical and electronic systems. Routledge.

Du, X., Nagose, A., Bloom, A., \& Julson, T. (2017). Prognosis of connector disconnection using a canary-based approach. In Proceedings of the annual conference of the prognostics and health management society.

Eki, H., Teratani, T., \& Iwasaki, T. (2007). Power consumption and conversion of eps systems. In Proceedings of the power conversion conference. 
Furse, C., Chung, Y. C., Dangol, R., Neilson, M., Mabey, G., \& Woodward, R. (2003). Frequency-domain reflectometery for on-board testing of aging aircraft wiring. IEEE Transctions on Electromagnetic Compatibility, 45, 306-315.

Furse, C., Chung, Y. C., Lo, C., \& Pendalaya, P. (2006). A critical comparison of reflectometry methods for location of wiring faults. Smart Structures and Systems, 2, 25-46.

Hwang, K.-Y., \& Kwon, B.-I. (2019). Design of low-cost blac motors for integrated electric brake systems. IEEE Access, 7, 184183-184193.

Lin, W.-C., \& Du, X. (2018). Prognosis of power connector disconnect and high resistance faults. In Proceedings of the 2018 IEEE International Conference on Prognostics and Health Management.

Lin, W.-C., \& Ghoneim, Y. A. (2016). Model-based fault diagnosis and prognosis for electric power steering systems. In Proceedings of the 2016 IEEE International Conference on Prognostics and Health Management.

Mohtat, A., Garner, G., Lin, W.-C., \& Mehrabi, N. (2020). Validation and refinement of a steering friction increase detection algorithm using test drive data. In Proceedings of the annual conference of the prognostics and health management society.

Morton, C., Spargo, C. M., \& Pickert, V. (2014). Electrified hydraulic power steering system in hybrid electric heavy trucks. IET Electrical Systems in Transportation, 4(3), 70-77.

Okada, T., Nishina, S., Ataka, T., Hashimoto, M., Irisawa, A., \& Imamura, M. (2015). Development of terahertz pulse time-domain reflectometry system for transmission line failure analysis. In Proceedings of the 40th international conference on infrared, millimeter, and terahertz waves.

Shi, Q., \& Kanoun, O. (2014). A new algorithm for wire fault location using time-domain reflectometry. IEEE Sensors Journal, 14, 1171-1178.

Smail, M. K., Hacib, T., Pichon, L., \& Loete, F. (2011). Detection and location of defects in wiring networks using time-domain reflectometry and neural networks. IEEE Transactions on Magnetics, 47, 1502-1505.

Smail, M. K., Pichon, L., Olivas, M., Auzanneau, F., \& Lambert, M. (2010). Detection of defects in wiring networks using time domain reflectometry. IEEE Transactions on Magnetics, 46, 2998-3001.

Smith, P., Furse, C., \& Gunther, J. (2005). Analysis of spread spectrum time domain reflectometry for wire fault location. IEEE Sensors Journal, 5, 1469-1478.

Tsai, P., Lo, C., Chung, Y. C., \& Furse, C. (2005). Mixedsignal reflectometer for location of faults on aging wiring. IEEE Sensors Journal, 5, 1479-1482.

Will, B., \& Rolfes, I. (2013). Comparative study of moisture measurements by time domain transmissometry. IEEE
Sensors Journal, 1-4.

\section{BIOGRAPHIES}

Wen-Chiao Lin received the M.S.E. and Ph.D. degrees in Electrical Engineering: Systems both from University of Michigan, Ann Arbor, MI. From 2007 to 2013 he was with Idaho National Laboratory, where he participated in research projects on developing advanced online anomaly detection and control technologies for large facilities, in which large amounts of sensors are deployed and may be unreliable or subject to cyber attacks. He is currently with General Motors Global R\&D, where he develops fault diagnosis, prognosis, and mitigation algorithms as well as fault tolerant control strategies for vehicle systems. His research interests are in the areas of systems science and control theory and their applications to engineering systems. He is the author or co-author of more than 30 peer-reviewed publications and more than 25 issued or pending US Patents.

Graeme Garner received the B.S.E. from Queen's University, Ontario, Canada, in 2018, where he studied in a dual program of Applied Mathematics and Mechanical Engineering. He is the recipient of the J.B. Stirling Gold Medal for graduating with the highest academic standing in his class and the Keyser prize for his research project on adapting Qlearning to decentralized stochastic control problems. His diverse professional background includes developing oil production forecasting algorithms at the Alberta Energy Regulator and studying automated trading strategies at the Canadian Imperial Bank of Commerce. He is currently at the Canadian Technical Center of General Motors, where he develops prognostics algorithms for vehicle hardware. His research interests are in machine learning and control theory, and their intersection in intelligent vehicle systems.

Yat-Chung Sunny Tang received the B.S.E.E and M.S.E.E from Oakland University, Rochester, Michigan. He is currently the Technical Specialist for Controlled Steering at General Motors Vehicle Motion Embedded Controls team. He is responsible for the hardware/software design and development of the steering electronic control unit, sensor, and actuator globally. Sunny has over 27 years of experience in various technical positions in the automotive industry. He is the author or co-author of 3 peer-reviewed publications and 4 issued or pending US Patents. He is very excited about the path the automotive industry is following when moving into the new era of mobility.

Arash Mohtat received his B.Sc. and M.Sc. from Univ. of Tehran in 2007 and 2009; and, his Ph.D. from McGill University in 2014 . From 2015 to early 2017, he was a postdoctoral fellow at McGill University working on multi-modal human computer interfaces as well as a research project that spun off into Haply Robotics: a start up with the mission of democratizing haptics and robotics technologies. From 2017 to 2020, Arash was with the Canadian Technical Center of General Motors leading multiple vehicle health management projects. Since 2020, Arash has been a technical product manager at Invision AI architecting smart infrastructure solutions. Arash has very diverse research interests with a key central theme: mechatronic systems with different levels of intelligence and autonomy. As a researcher/engineer with entrepreneurial enthusiasm, he has several peer-reviewed publications and pending US Patents. 


\section{APPENDIX}

The following maneuvers are included in the test data. Each numbered list of actions describes a single test instance. The quantity of tests with each maneuver is included in parenthesis next to the test name.

\section{Lane Changes (23):}

1. Cruise at $25 \mathrm{mph}$;

2. 2 single lane changes;

3. 2 double lane changes;

4. Cruise at $50 \mathrm{mph}$;

5. Repeat 2-3.

\section{Turns (41):}

1. $\frac{1}{4}$ circle at $30 \mathrm{mph}$, one in each direction;

2. Two $90^{\circ}$ turns from stop in each direction;

3. One U-turn in each direction at $10 \mathrm{mph}$.

\section{Spirals (8):}

1. $\frac{1}{4}$ circle at $30 \mathrm{mph}$, one in each direction;

2. Two $90^{\circ}$ turns from stop in each direction.

\section{Sinusoidals (41):}

1. Cruise at $30 \mathrm{mph}$;

2. 4 low-amplitude sinusoidals (using about 1 lane width);

3. 4 medium amplitude sinusoidals (using two full lanes);

4. Repeat $2-3$ at $50 \mathrm{mph}$;

5. Repeat $2-3$ at $60 \mathrm{mph}$.

Organics (36):

1. 13 minute normal driving at posted speed limits.

Noise factors:

Table 8 documents the noise factors introduced in test data.

Table 8. Data collection noise factors.

\begin{tabular}{|l|l|}
\hline Noise factor & Varied levels \\
\hline Tire type & All-season, all-terrain, and winter \\
\hline Tire pressure & 20,30, and 40 psi \\
\hline Battery load & Rear defrost on and off \\
\hline Passenger load & 2,4, or 6 passengers \\
\hline Surface conditions & Wet, dry \\
\hline
\end{tabular}

